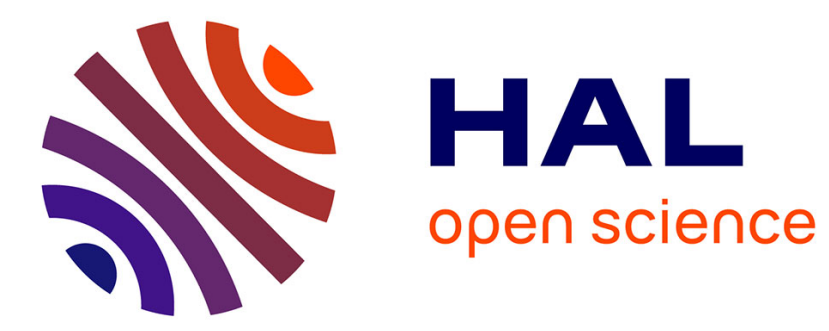

\title{
On the equivalence of unidirectional rogue waves detected in periodic simulations and reproduced in numerical wave tanks
}

Guillaume Ducrozet, Félicien Bonnefoy, Pierre Ferrant

\section{To cite this version:}

Guillaume Ducrozet, Félicien Bonnefoy, Pierre Ferrant. On the equivalence of unidirectional rogue waves detected in periodic simulations and reproduced in numerical wave tanks. Ocean Engineering, 2016, 117, pp.346-358. 10.1016/j.oceaneng.2016.03.027 . hal-01299500

\section{HAL Id: hal-01299500 \\ https://hal.science/hal-01299500}

Submitted on 7 Apr 2016

HAL is a multi-disciplinary open access archive for the deposit and dissemination of scientific research documents, whether they are published or not. The documents may come from teaching and research institutions in France or abroad, or from public or private research centers.
L'archive ouverte pluridisciplinaire HAL, est destinée au dépôt et à la diffusion de documents scientifiques de niveau recherche, publiés ou non, émanant des établissements d'enseignement et de recherche français ou étrangers, des laboratoires publics ou privés. 


\title{
On the equivalence of unidirectional rogue waves detected in periodic simulations and reproduced in Numerical Wave Tanks
}

\author{
Guillaume Ducrozet*, Félicien Bonnefoy, Pierre Ferrant \\ LHEEA Lab., Ecole Centrale Nantes, CNRS, Nantes, France
}

\begin{abstract}
This paper deals with the reproduction of unidirectional extreme events in a numerical wave basin. From a rogue wave measurement at a given location, experiments or numerical simulations are conducted with the same wave profiles using reproduction procedures. Although it is recognized that many different physical mechanisms may be at play in freak wave formation, reproduction procedures generally use frequency focusing to generate these high waves. This paper intends to assess the validity of this approach. In particular, it will focus on the relationship between an accurate reproduction of the free surface elevation and the accuracy of the corresponding wave kinematics inside the fluid domain. A highly nonlinear model is used to compute the occurrence of unidirectional freak waves in large periodic domain, which are reproduced in a 2-D Numerical Wave Tank and compared. The study presents an advanced reproduction procedure that deals with rogue waves
\end{abstract}

\footnotetext{
${ }^{*}$ Corresponding author.

E-mail address: guillaume.ducrozet@ec-nantes.fr

Tel: +33240371645

Fax: +33240372523
} 
embedded in an irregular sea state. It shows that, using the appropriate reproduction procedure, it is possible to accurately reproduce free surface elevation as well as kinematics induced by this extreme event.

Keywords: Extreme waves, Wave focusing, Non-linear wave interactions, High-Order Spectral method, Numerical Wave Tank, Reproduction, Wave kinematics

\section{Introduction}

In the open ocean, a wide variety of sea conditions may be encountered. Marine structures must be designed to withstand, even the most extreme wave fields. In this context, the existence of freak or rogue waves is now acknowledged as a possible threat for large ships or any marine structure. The New Year's wave, observed in North Sea, is the first to have been monitored and reported [22]. It has become a reference event for a large number of studies, representing a realistic extreme event for the analysis of the response of structures to such waves, e.g. see experiments in [10]. Note that other freak waves have been monitored and reported, see the review in [25] for instance.

Wave-structure interactions are typically analyzed through experiments or numerical simulations. However, it is not always obvious how to consider the interactions with rogue waves. One possible approach is to consider a given wave field that is known to produce extreme events (by means of focusing, Benjamin-Feir instability, see e.g. [5, 45, 37]). Otherwise, the study of these interactions usually relies on the reproduction of a given measured event at sea, usually time series obtained from a point sensor installed in the ocean. From these local measurements, a complete wave field is reconstructed 
to coincide with data at a given location. Procedures to accurately reproduce the measured wave profile have been developed, initially in the context of wave basins, but may also be applied to Numerical Wave Tanks (NWTs). The simplest approach is the use of frequency focusing, assuming waves are linear and unidirectional; more complex procedures attempt to account for wave-wave interactions (see [20] for a review of classical methods for wave reproduction; and $[40,32]$ for development of a promising new approach).

Note that some observations with spaceborne Synthetic Aperture Radars (SARs) are now available, offering a directional information on the wave field. However, it is for now mainly used as a source of statistical information about the wave field i.e. to determine directional wave spectra (see [26] for instance). To assess the accuracy of such a system in the description of 3-D free surface elevation in a given sea state and consequently use it to measure/detect extreme events, extensive validations are still needed.

Thus, classical reproduction algorithms always use the dispersive character of gravity waves to create the extreme events by spatio-temporal focusing in unidirectional wave field (with possible adjustments due to nonlinear effects). However, focusing (spatio-temporal or directional) is only one of several physical mechanisms that play a key role in the formation of rogue waves, see e.g. $[24,29]$. Others include non-linear wave interactions (modulational instabilities), wave current interactions, atmospheric forcing, etc.

This paper evaluates the validity of this approach (reproduction with focusing), and allows good reproduction of rogue waves with respect to free surface elevation as well as wave kinematics inside the fluid domain. This 
is a key point in the analysis of the interaction of these extreme waves with marine structures. The latter are up to now always based on 2-D (i.e. unidirectional) wave fields for simplicity of experimental or numerical set-up. Consequently, the present study is limited to unidirectional sea states. However, note that the directional spreading is important and essential to nonlinear physics (including rogue waves), see e.g. [21, 36, 41].

As stated earlier, data collected about rogue waves are limited to time series of free surface elevation obtained from a sensor at a given location. Thus assessment of the accuracy of the reproduced event can only be based on these data. Consequently, we have chosen to adopt a numerical approach to treat this problem. However, freak waves exhibit large amplitudes, high steepness and short duration, making their simulation still challenging. Indeed, the use of time-domain highly-nonlinear potential models appears to be the most suitable approach for studying such extreme events. Note that we limit our approach to unidirectional waves evolving in finite constant depth without wind or current.

The non-linear potential model chosen is based on the High-Order Spectral (HOS) method proposed by West et al. [42] and Dommermuth \& Yue [13]. This method has been validated on several test cases, which have demonstrated its efficiency and accuracy, making it suitable for large scale simulations. It has also been applied to modeling of freak waves in [15], allowing us access to all information required for the description of the original extreme events: free surface elevation, induced kinematics, etc. Several physical phenomena have been studied thanks to this highly nonlinear model, 
e.g. non-linear energy transfers [35], modulational instabilities [19, 38], and bi-modal seas [39] among others.

From the extracted temporal signal, a reproduction procedure is set up with a Numerical Wave Tank. In this paper, we intend to reproduce the extreme event, embedded in an irregular wave field, which reflects the conditions under which actual rogue waves appear. The wavemaker motion is deduced using non-linear phase velocity [27] and possible iterations are conducted for correcting the remaining phase shift. The NWT used is also based on the HOS scheme, but adapted to take into account a wave generator, reflective walls and an absorbing beach, leading to the HOS-NWT model [16]. This model has already been widely validated for free surface elevation prediction as well as wave induced kinematics, with several comparisons to experiments on different sea states: from regular unidirectional waves to directional irregular sea states. Consequently, comparisons of all physical features of the 'natural' freak wave and the reproduction will be available.

In Section 1, the nonlinear potential flow models will be briefly presented in their two configurations: periodic domain and NWT. In Section 2, the embedded reproduction procedure used is discussed and applied to the wellknown New Year's wave for validation. In the last part, a large periodic domain is simulated thanks to the HOS model and different freak waves are detected in this configuration. From the corresponding wave signals, the reproduction procedure is applied and both extreme events in open periodic domain and in the wave basins are compared with specific attention paid to 
the pressure and velocity fields at the moment of focusing.

\section{Nonlinear wave models}

This section presents the non-linear wave models used in the following to simulate a rogue wave appearing in periodic (open) domain (HOS-ocean) and the NWT used for the reproduction (HOS-NWT). These are based on the HOS method proposed by West et al. [42] and Dommermuth \& Yue [13]. Both models use the same core but have different initial and boundary conditions. HOS-ocean computes the evolution of a given initial wave field in a large periodic domain while HOS-NWT simulations start from rest with the waves generated thanks to a wavemaker, propagated in the closed domain (reflective walls) and possibly absorbed. More details on both methods (detailed description, validation, etc.) can be found in [15] \& [16] for HOS-ocean \& HOS-NWT respectively. Note that both models are now available as open source $[2,1]$. The methods are presented for the 2 -D problem, but extension to $3-\mathrm{D}$ is straightforward, see $[15,16]$.

\subsection{Formulation}

We consider a rectangular fluid domain $D$ of horizontal dimension $L_{x}$ and constant water depth $h$ associated with a Cartesian coordinate system. Its origin $O$ is located at one corner of the domain with $O x$ axis representing the horizontal axis and $O z$ the vertical axis oriented upward with $z=0$ located at the mean free surface. We are working under the potential flow theory (assuming the fluid to be incompressible and inviscid and the flow irrotational). Under these assumptions, the continuity equation reduces to the Laplace equation for the velocity potential $\phi$. 
Boundary conditions close the system of equations. Lateral boundary conditions are specific to the problem solved (open periodic domain in Sec. 1.2, NWT in Sec. 1.3). However, in both cases, in addition to bottom boundary condition, they allow us to define a spectral basis on which the velocity potential in the whole volume will be expanded. At the same time, surface quantities are also expressed on a spectral basis allowing the use of Fast Fourier Transforms (FFTs). $z=\eta(x, t)$ describes the free surface position, assuming no wave breaking occurs. We consider the non-linear free surface boundary conditions (kinematic and dynamic) which are written, following [44], using surface quantities $\eta$ and $\widetilde{\phi}(x, t)=\phi(x, z=\eta(x, t), t)$ the free surface velocity potential.

These non-linear free surface boundary conditions allow to advance in time the two unknowns $\eta$ and $\widetilde{\phi}$, once the so-called HOS method evaluates the unknown vertical velocity. The HOS procedure relies on a series expansion in wave steepness $\epsilon$ up to the so-called HOS order $M$ of the velocity potential. Expanding a Taylor series around $z=0$ and collecting terms at each order in wave steepness leads to a triangular system. A similar series expansion for the vertical velocity leads to another triangular system, which is solved iteratively.

The resulting numerical method is pseudo-spectral and exhibits very interesting convergence properties. Thus, this HOS model features high efficiency and accuracy compared to other advanced methods for wave propagation, see [14]. 


\subsection{HOS-ocean}

HOS models have been widely used for the study of wave propagation in open domains starting from the original work of [42] \& [13] up to the analysis of complex wave phenomena [35, 38, 39, 19], including freak waves [15, 43]. In this model, periodic lateral boundary conditions are used, assuming the domain to be infinite. This fixes the choice of spectral basis used to represent variables and enables the use of FFTs to evaluate Fourier components from a spatial description (direct or inverse).

With such an approach, the key point lies in the definition of an appropriate initial condition. The initial free surface elevation and free surface velocity potential must be determined before propagating the corresponding wave field in the HOS-ocean model. It has been demonstrated, in [12], that the definition of linear initial wave fields for fully nonlinear computations can lead to numerical instabilities. In order to avoid this problem, a transition period is introduced through the use of a relaxation scheme, allowing a smooth transition from linear initial conditions (wave components extracted from wave spectrum) to fully nonlinear computation.

We reiterate that several validations have been conducted that have demonstrated the accuracy and efficiency of the model. We refer to the different publications cited at the beginning of the section for details.

\section{3. $H O S-N W T$}

When dealing with a physical wave basin, waves are generated with a wavemaker located at one end of the basin, starting from rest. This configuration is specific and quite different from that presented in previous section. 
Thus, the need for a dedicated model has led to the development of a $\mathrm{Nu}$ merical Wave Tank, namely HOS-NWT, which is able to reproduce both the wave generation process and their propagation in a wave basin.

In contrast to the HOS-ocean model, HOS-NWT considers the lateral walls to be perfectly reflective. This change in boundary conditions leads to another set of basis functions. However, this choice still enables the use of FFTs in the method for the transformations between Fourier and physical space (back and forth).

The initiation of waves by the wavemaker movement introduces an inhomogeneous condition at the wavemaker position $x=0$. A specific procedure has been developed for an efficient and accurate description of the wavemaker. The main idea, following [4], is to introduce the concept of additional potential. The potential $\phi$, solution of the whole problem (generation and propagation), is separated into two parts: $\phi=\phi_{\text {spec }}+\phi_{\text {add }} . \phi_{\text {spec }}$ is the previously described spectral potential in the fixed geometry tank with its free surface, and $\phi_{a d d}$ is the additional potential only accounting for the wavemaker. In addition, an absorbing zone is modeled in the numerical model through a local modification of the free surface dynamic boundary condition. We refer to [16] for all details.

Specific attention has been paid to preserving the pseudo-spectral formalism of this complete NWT (including wave generation, see [16]). This way, it has been shown to achieve the same level of efficiency and accuracy as HOSocean. Several validations have been undertaken, notably some comparisons of free surface elevation and/or pressure fields with experiments conducted 
in the Ecole Centrale Nantes $(\mathrm{ECN})$ wave basin $(50 m \times 30 m \times 5 m)$, including complex 2-D and 3-D wave fields (regular and irregular waves, directional focusing), see [16].

\section{Reproduction procedure}

Measurements of extreme events in open ocean are usually limited to free surface elevation at a single location. This kind of measurement is widely used and has revealed the existence of rogue waves in ocean. The most famous such wave is probably the New Year's wave (or Draupner wave) recorded on January $1^{\text {st }}, 1995$ at the Draupner platform in the North Sea [22]. This freak wave measured in real conditions has been widely studied as a reference event $\left(H_{\max }=25.6 \mathrm{~m}\right.$ occurring in a wave field characterized by a significant wave height $\left.H_{s}=11.9 \mathrm{~m}\right)$. Specific efforts were undertaken in order to try to understand its physics and, since rogue waves are of particular importance for ocean and naval engineering, different experimental campaigns have been initiated to study the impact of such extreme waves on marine structures $[10,30]$.

It is evident that there is a need to reproduce as accurately as possible a free surface elevation signal recorded in open ocean in a wave basin. To this end, various reproduction procedures have been developed, especially in the context of physical wave basins, see e.g. $[8,11,31,20]$. In this section, we present a procedure applied to HOS-NWT that aims to reproduce an extreme event embedded in an irregular wave field, which reflects the real conditions in which rogue waves appear.

Note that the same kind of procedure may be used in physical wave basins: 
the various validations comparing HOS-NWT to experiments conducted in ECN wave basin have shown that this is an accurate numerical method [16]. Furthermore, our physical and numerical wave tanks use the same description of wavemaker motion, making the procedure presented hereafter easily transferable to physical wave basins.

We focus on the New Year's wave [22]. The top chart in Fig. 1 presents the free surface elevation measured at the Draupner platform as a function of time. In Section 2.1, we present in detail the approach used for the reproduction, and in Section 2.2 the tools used for the estimation of its quality . In Section 2.3, results obtained from the reproduction of this specific extreme event are discussed.

\subsection{Embedded reproduction scheme}

The reproduction of a large event generally makes use of the dispersive character of gravity waves. That is to say, the extreme event is intended to be reproduced by frequency focusing, as presented e.g. by Chaplin [8]. In addition, interesting enhancements or alternatives have recently been developed, see $[11,32,20]$. Different frequencies with a given energy are generated so that they are in phase at the position and time of the focusing $\left(x_{f}\right.$ and $t_{f}$ respectively). The amplitude and phase of each frequency are then obtained using linear theory, starting from the target signal one wants to reproduce.

The approach chosen here is to embed the extreme event inside the irregular wave field that exists at the moment of focusing, as in [34]. This irregular sea state can be characterized by the temporal signal containing

the freak wave, as presented in Fig. 1. The generation of large waves within 
an irregular wave field may be used for ship model testing, see e.g. [33, 34]. The main difference here with [34] is that we seek to control the shape of the generated extreme event.

The first step is to choose the duration of the time window we want to reproduce as well as the position of focusing $x_{f}$. The time-window is chosen sufficiently long (typically $\simeq 50 T_{p}$ in the different tests presented here) to contain a sufficiently representative description of the frequency components of the reproduced wave field. Figure 1 presents an example of a time-window chosen for reproduction. A ramp is also applied on the signal to make it periodic and suitable for frequency analysis.
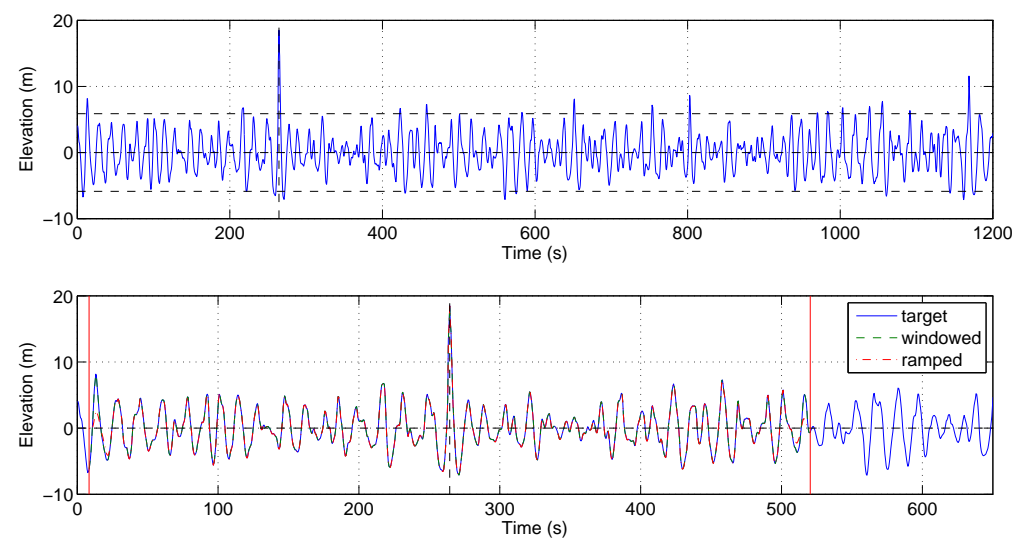

Figure 1: Time history including the freak wave measured at the Draupner platform on January $1^{\text {st }}, 1995$ (top) and time-window chosen for reproduction (bottom)

Next, the frequency content of the target time signal has to be truncated at a maximum frequency $f_{\max }$ which defines, with $x_{f}$, the minimum focusing time $t_{f}$ (using the linear group velocity $\left.c_{g}: t_{f}=\frac{x_{f}}{c_{g}\left(f_{\max }\right)}\right)$. Note that the 
frequency cut-off has to be chosen such that it does not alter the target wave.

Using linear theory, it is possible to propagate this wave field backwards from the focusing position $x_{f}$ up to the desired wavemaker position (chosen by convention as $x=0$ ) and to deduce its motion. In order to embed the reproduction inside an irregular wave field, the back-propagation is realized in such a way that the generation is taking place over a sufficiently long duration. The idea is that when the shortest waves (part of the extreme event) are generated, they are propagating in a wave field containing all other frequency components. In this way, the wavemaker motion is similar to what may be seen for the generation of an irregular wave field, as presented in Fig. 2. Vertical lines indicate the repeat period of the wavemaker motion, each sequence (lasting $512 \mathrm{~s}$ ) will produce an extreme wave, but only the second one can be considered to be embedded in the irregular sea from its generation. We point out that in order to ensure high accuracy, the motion of the wavemaker is evaluated so that free waves generated by it can be controlled (and suppressed), see [7] for details.

Generation and propagation of such the considered wave field would be expected to cause a phase shift between the reproduced event and the target wave. This is due to nonlinear effects resulting in a change of wave speed for each frequency component. Thus, the discrepancies between two signals will be more important when $x_{f}$ is larger. However, in wave basin tests there is a lower threshold for $x_{f}$ beyond which this focusing location cannot be further reduced in order to avoid the influence of the evanescent modes existing close to the wavemaker (decaying as $\left.\exp \left(-k_{p} x\right)\right)$. 


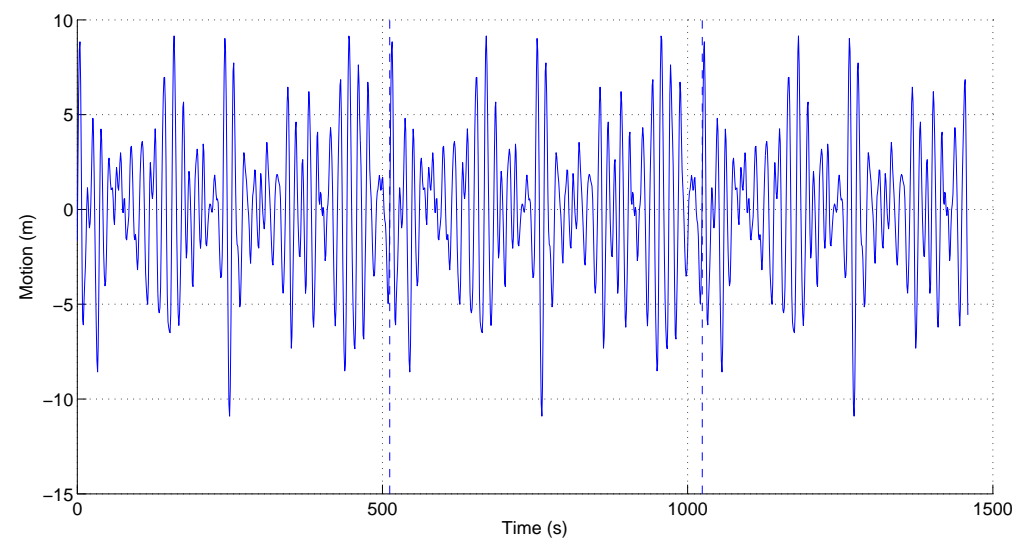

Figure 2: Wavemaker motion for Draupner wave reproduction

Since we are considering the case of the propagation of an extreme wave embedded in an irregular sea, we can consequently assume that the propagation takes place in an homogeneous state. Thus, it is possible to estimate a theoretical phase shift following the third-order solution of Madsen \& Fuhrman [27]. The latter publication provides an expression of the thirdorder phase velocities for an irregular wave field in finite depth. This is used to determine a theoretical phase shift between linear (i.e. target) and nonlinear (i.e. reproduction) wave fields at a given location $x_{f}$. This may be used to correct the wavemaker motion, which had initially been determined assuming linear theory.

Previous studies dealing with reproduction procedures have already tried to correct the phasing of the different components iteratively [8, 20]. After the theoretical correction presented previously, such a procedure is also applied in our reproduction scheme. 
For an accurate phase adjustment, we use the decomposition of odd and even nonlinear terms, as used in Johannessen \& Swan [23]. The procedure relies on the generation of a second wave field with components of the same amplitude than the first wave field but with a $\pi$-phase shift applied. The first generated wave field containing the extreme event presented before (with free surface elevation $\eta(t)$ ) may be seen as a crest focusing, while the second is a trough focusing $\left(\eta_{\pi}(t)\right)$. From those two simulations, we construct the odd and even free surface elevation:

$$
\begin{aligned}
\eta_{\text {odd }} & =\left(\eta-\eta_{\pi}\right) / 2 \\
\eta_{\text {even }} & =\left(\eta+\eta_{\pi}\right) / 2
\end{aligned}
$$

The odd free surface elevation contains the first (and third, fifth... ) components, while the even free surface elevation contains the second (and fourth, sixth...) components. This way, we extract the second order terms of the analysis, using $\eta_{\text {odd }}$ for the determination of phase shift correction and new wavemaker motion.

Note that it is also expected that a possible discrepancy in wave amplitudes may be experienced and attributable to the non-linear interactions that enable the exchange of energy between different components (four-wave interactions). However, as noted in [20], an exact control of the amplitude at the position of focusing seems very difficult to achieve in such irregular wave fields, even if some recent attempts have been made [31, 20]. Thus, we restrict the adjustment procedure hereafter to phase corrections. 


\subsection{Performance estimation}

In order to evaluate the accuracy of the reproduction procedure with respect to the target wave, different comparisons will be provided. The first one concerns the temporal signal at the position of the focusing (see e.g. Fig. $3)$.

Then, to improve the analysis, a similar comparison between target and reproduced waves is provided in the frequency domain. An example may be seen in Fig. 4. The top chart presents the amplitudes of both waves in the frequency domain, while the bottom chart depicts phase shift between the target and the reproduced wave field at the focusing position $x_{f}$. For the phase shift, when appropriate, the theoretical solution of the third order solution [27] will be depicted.

These comparisons do not allow the error made on the reproduction to be quantified. Therefore, an estimation of the accuracy is provided by evaluating a correlation coefficient defined as:

$$
R_{\eta}\left(\eta_{t}, \eta_{r}\right)=\frac{C\left(\eta_{t}, \eta_{r}\right)}{\sqrt{C\left(\eta_{t}, \eta_{t}\right) \cdot C\left(\eta_{r}, \eta_{r}\right)}}
$$

where function $C$ is the covariance, $\eta_{r}$ the reproduced signal and $\eta_{t}$ the target signal. In the following, this correlation coefficient is evaluated on a timewindow of length $4 T_{p}$ centered on the extreme event.

For the final choice of the accurate reproduction (i.e. when $R_{\eta}$ is sufficiently large), the amplitude of the extreme event is also an important parameter. The accuracy with respect to this crest amplitude is quantified 
by a second coefficient:

$$
R_{c}=\frac{\max \left(\eta_{r}\right)}{\max \left(\eta_{t}\right)}
$$

\subsection{Results on New Year's wave}

As stated earlier, 3-D character of the sea state is essential to non-linear processes, including rogue waves. As a consequence, the New Year Wave has been shown to be too steep to simulate in uni-directional waves [9]. Furthermore, it has been argued that there is strong evidence that it occurred in a bi-directional sea [3]. However, following previous studies (e.g.[11]), this real measurement at sea appears as an appropriate validation of the reproduction procedure.

In this section, we present the results of applying the previously described reproduction procedure to the New Year's wave [22] with HOS-NWT. The numerical parameters chosen are the following

- Domain length: $L_{x}=6000 \mathrm{~m}$

- Water depth: $h=70 \mathrm{~m}$

- HOS order: $M=3$

- Discretization: $N_{x}=769$ points/nodes (i.e. 1537 dealiased modes) and $N_{z}=33$ points for the wavemaker

- Duration of simulation: $T=1500 \mathrm{~s}$

Figure 3 presents the results of the reproduction procedure in the time domain without any phase correction. As expected, the temporal signal exhibits a phase shift between the target wave and its reproduction. From 
the top chart of Fig. 3 it can be observed that the extreme event appears as part of an irregular sea. Due to its phase shifting, the correlation coefficient is consequently rather low: $R_{\eta}=0.26$. In order to characterize this phase shift, the frequency analysis is provided in Fig. 4.
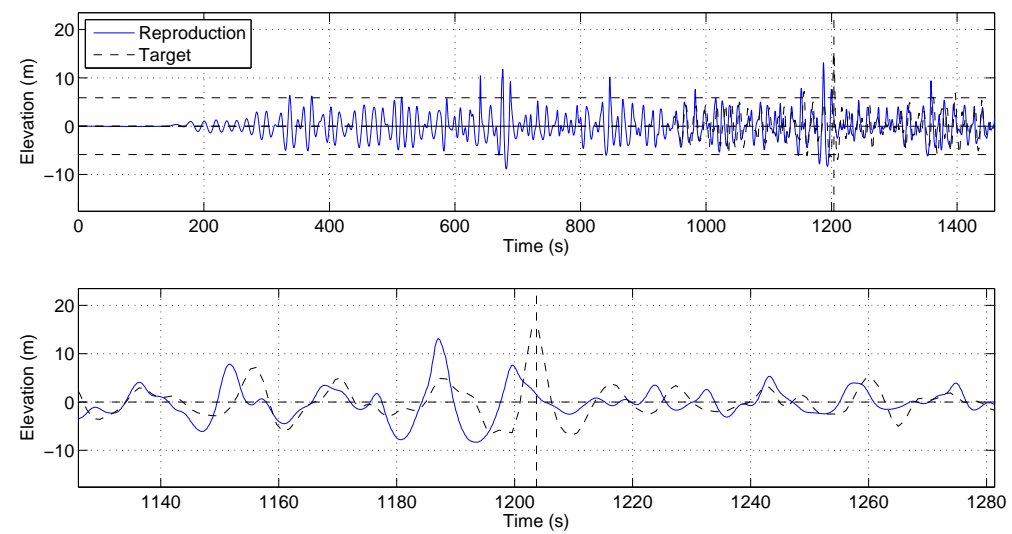

Figure 3: Comparison between reproduction and target wave (time domain) without any phase correction, $R_{\eta}=0.26$

First, we focus on the observed difference in amplitude of frequency components between the target and reproduced waves. These occur during propagation and may be attributed to the non-linear interactions, which enable the exchange of energy between different components (four-wave interactions).

This frequency analysis confirms the possibility of correcting the phases of the wavemaker motion using third-order theory, as presented in [27]. Indeed, this theoretical phase shift agrees well with that measured between the target signal and its numerical reproduction in HOS-NWT (see bottom of Fig. 4). This means that the non-linear interactions are responsible for the main discrepancies observed if no correction is applied. Note that the 

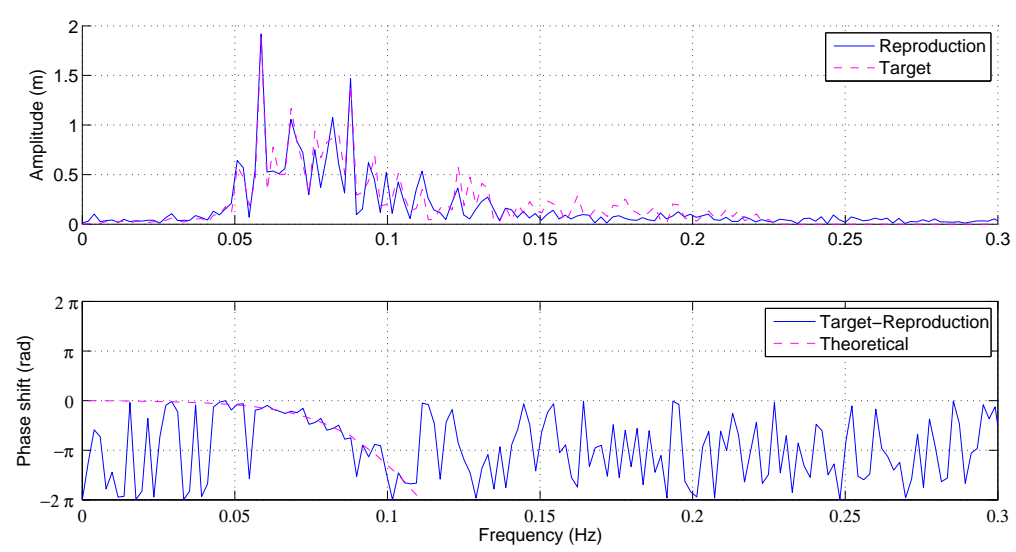

Figure 4: Comparison between reproduction and target wave (frequency domain)

extreme event must be embedded in an irregular wave field for the correction to be valid (i.e. homogeneous sea-state).

Figures $5 \& 6$ present the results obtained when the phases of the wavemaker motion are adjusted with respect to third-order theory. Note that after this theoretical phase correction, same kind of differences in terms of amplitude of frequency components is observed than in Fig. 4 and is not represented here for clarity.

We can observe an enhancement of the phasing between reproduction and target waves; the correlation coefficient is now $R_{\eta}=0.70$. The frequency analysis (Fig. 6) demonstrates that the proposed correction has eliminated most of the phase shift observed with the first simulation. However, since the physical mechanisms at play are highly non-linear, this phase correction is often not sufficient, and successive iterations are run in order to achieve a satisfactory reproduction. 


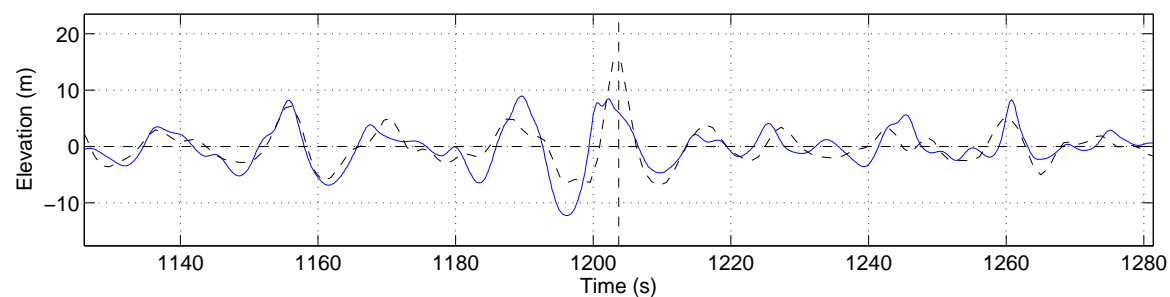

Figure 5: Time-domain comparison between reproduction and target wave with theoretical phase correction, $R_{\eta}=0.70 . T_{r}=T_{\text {repeat }}=512 s, x_{f}=10 \lambda_{p}$

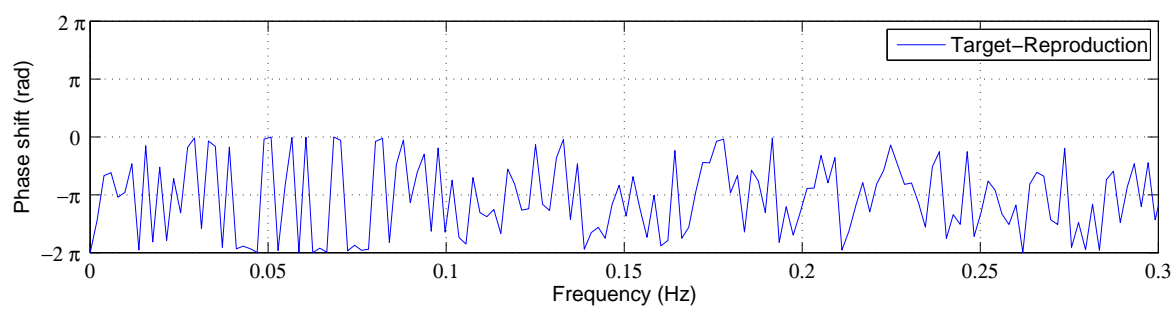

Figure 6: Frequency-domain comparison between reproduction and target wave with theoretical phase correction. $T_{r}=T_{\text {repeat }}=512 s, x_{f}=10 \lambda_{p}$ 
The set-up of the quality estimator $R_{\eta}$ is then interesting for assessing if an iteration is satisfactory or not. This gives a fair estimate of the accuracy of the reproduction procedure, with respect to both the extreme event and the surrounding waves (one may adjust the time window of correlation computation). The iterative process is consequently pursued and Fig. 7 presents an accurate result after five iterations, exhibiting a high correlation coefficient of $R_{\eta}=0.94$ and a corresponding crest amplitude ratio $R_{c}=0.85$. Note that, if needed, the iteration process can pay more attention to crest amplitude, see Sec. 3.2.

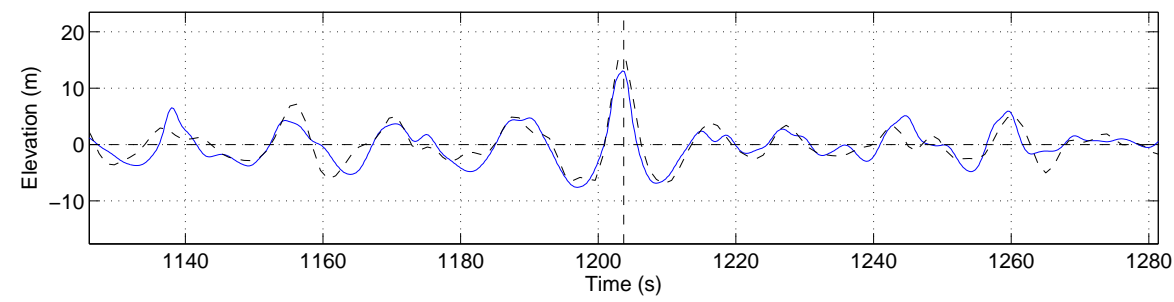

Figure 7: Iterative correction of phases, iteration 5, comparison with target, $R_{\eta}=0.94$, $R_{c}=0.85 . T_{r}=T_{\text {repeat }}=512 s, x_{f}=10 \lambda_{p}$

Therefore, we have developed an efficient method for the reproduction of extreme events, including freak or rogue waves in unidirectional wave fields. Its application to the well-known New Year's wave indicates its ability to reproduce such an event with good accuracy in terms of free surface profile. In the next section, we assess the reliability of reproduction schemes in greater details, applying the one presented to different rogue waves detected in simulations and paying specific attention to the kinematics of such highly nonlinear waves. 


\section{Application to a series of unidirectional rogue waves}

The previous section presented the reproduction scheme developed as an efficient procedure for 2-D highly non-linear waves. As already stated, the same procedure may be used in numerical and physical wave basins in order to accurately reproduce specific large events occurring in real seas and for which free surface measurements are available. In this section, we describe such reproduction procedures in more details and explore whether the ability to reproduce the free surface profile of a given wave is sufficient to capture all its physical features. The interaction of an extreme wave with a structure is mainly governed by the kinematics of the wave. Thus, we focus hereafter on these characteristics of freak waves and their reproduction. In real seas, free surface and velocity and/or pressures measurements are not usually available for such extreme events. Consequently, we propose a numerical analysis based on: i) simulation of the natural occurrence of different freak wave events in open domain using HOS-ocean, ii) the reproduction of these events with HOS-NWT and iii) detailed comparison of the kinematics for one specific event.

\subsection{Rogue waves in open domain simulations}

The HOS-ocean model can be used to simulate an irregular sea-state in a large periodic domain over long time periods. In this study, we restrict the analysis to 2-D computations (i.e. unidirectional wave field) in which the appearance of 'natural' freak waves is analyzed. Similar simulations in 2-D and 3-D have already been described in [15]. The considered water depth is $h=200 \mathrm{~m}$ and the initial condition is an irregular sea-state given 
by JONSWAP spectrum: $H_{s}=4 m, T_{p}=10 \mathrm{~s}, \gamma=3.3$. The numerical parameters are reported in the following

- Domain length: $L_{x}=82 \lambda_{p} \simeq 13 \mathrm{~km}$

- Duration of simulation: $T=2000 T_{p} \simeq 5 h 30 \mathrm{~min}$

- HOS order: $M=8$

- Discretization: $N=2048$ points/modes (i.e. 9216 dealiased nodes)

- Relaxation scheme for initialization: $T_{\text {relax }}=10 T_{p}$

A wave-by-wave analysis (in space) is performed during the simulation in order to extract the up-crossing wave heights inside the large domain at every time-step. The time evolution of the maximum wave height ratio $H_{\max } / H_{s}$ is presented in Fig. 8. The limit $H_{\max }=2.2 H_{s}$ is the conventional definition for a rogue wave.

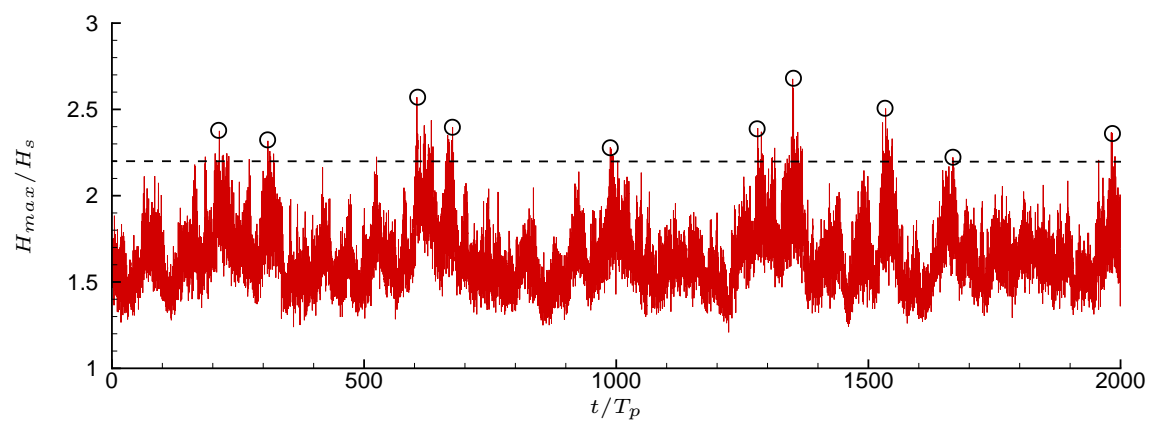

Figure 8: Time evolution of $H_{\max } / H_{s}$

From Fig. 8, we can observe that several freak waves appear during this long-time propagation. The circles identify the 10 different extreme waves 
that will be reproduced in the NWT. This will assess the capabilities of the presented scheme to reproduce different kind of waves in terms of free surface elevation. Then, one of the case is chosen for a detailed analysis of the induced kinematics inside the domain with comparison to the original freak wave observed in periodic open domain.

\subsection{Reproduction of different extreme events in a NWT}

Using HOS-NWT, we apply the reproduction procedure described in Sec. 2.1 to the different numerical probe signals obtained in the HOS-ocean simulation and containing the 10 identified freak events. Fig. 9 gives an example of signal for the 1 st freak wave detected in the domain. The HOS-NWT computations use domain length $L_{x}=3200 \mathrm{~m}$, HOS order $M=3$ and $N_{x}=512$ points/modes. This choice of numerical parameters allows to have an identical discretization in the periodic domain and the NWT simulations. This way, the effective spectral widths are the same. We choose a time window of $T_{r}=512 s \simeq 51 T_{p}$ and a focusing location $x_{f}=10 \lambda_{p}$.

For the different extreme waves, with no phase correction the behavior is similar to what has been observed in the case of the New Year's wave: a large phase shift is observed between the target and the reproduction. Consequently, theoretical phase correction was applied, followed by an iterative process to correct this phase error. With theoretical phase correction, the corresponding reproduction for the 1st freak wave is presented in Figs. 10 \& 11 in the time and frequency domains respectively. This reproduced event exhibits a correlation coefficient $R_{\eta}=0.55$ with the target extreme wave and $R_{c}=0.83$. 

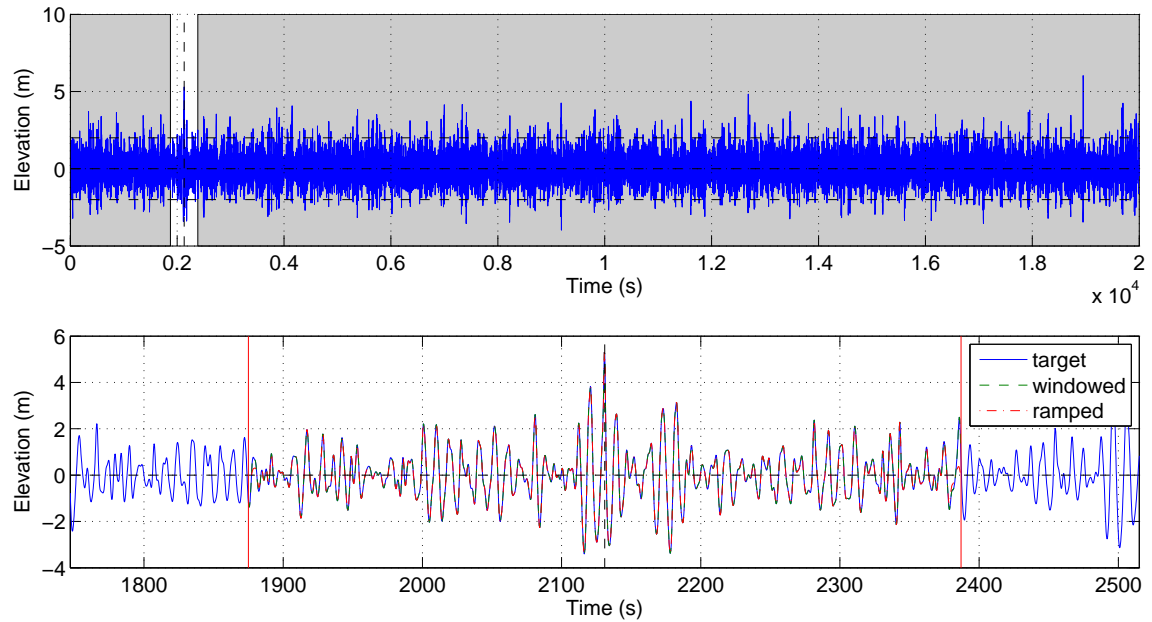

Figure 9: Time evolution of free surface elevation at the location of the freak wave (case 1).
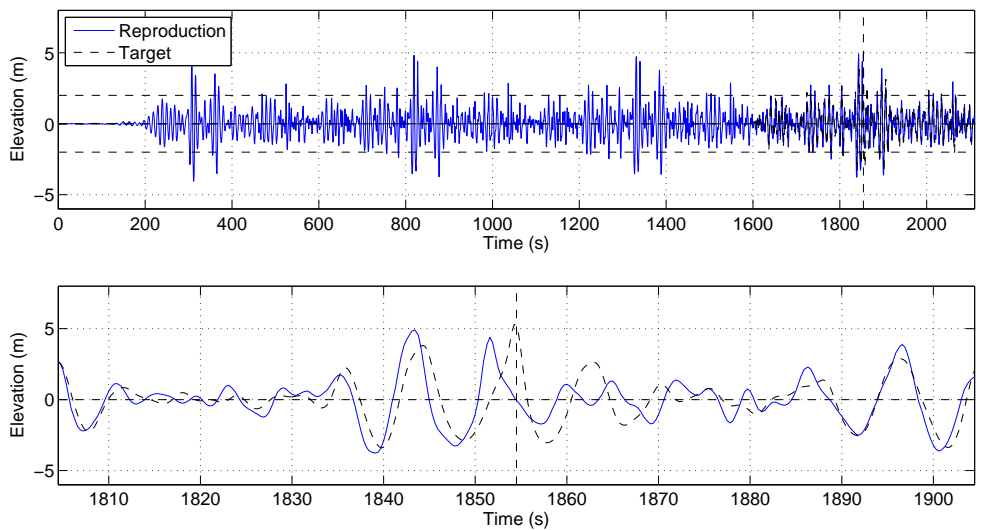

Figure 10: Comparison between reproduction and target wave (time domain) without iterative phase correction. $R_{\eta}=0.55, R_{c}=0.83$ 

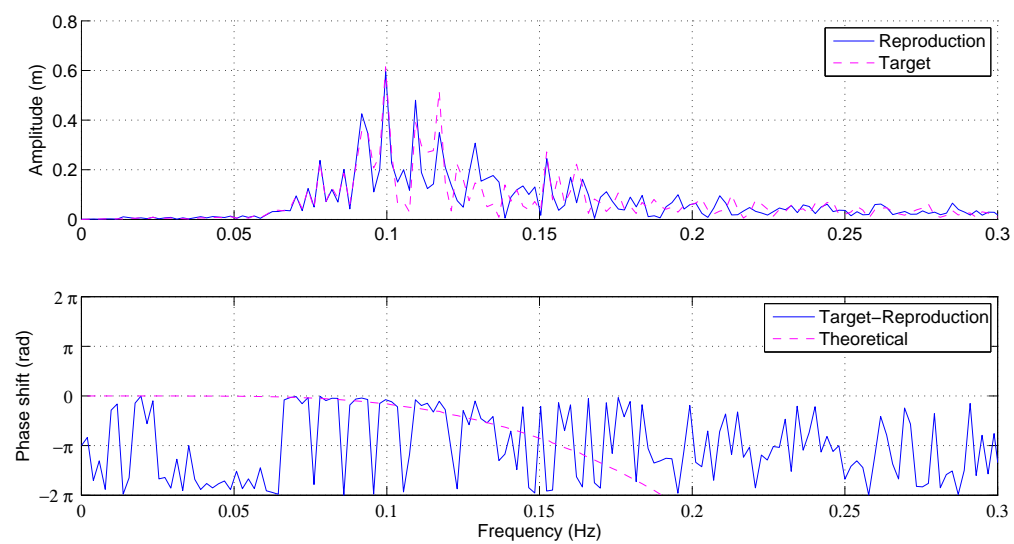

Figure 11: Comparison between reproduction and target wave (frequency domain)

The results after three iterations are presented in Fig. 12 for the same test case. Slight differences in the exact timing of maximal wave height can be observed, as well as some high frequency oscillations. The latter can be attributed to difficulties in controlling the phasing of all waves, particularly those of higher frequencies. The phase errors of high frequency components cause the observed oscillations. Despite these differences, we consider the extreme event to be satisfactorily reproduced, as assessed by the accuracy estimators $R_{\eta}=0.84$ and $R_{c}=0.95$.

Table 1 presents the results of the reproduction process for the 10 different extreme events identified in the open ocean numerical simulation. The characteristics of the original event as well as the accuracy estimators of the reproduced event are indicated.

At first, we note that the cases appearing with a star in Tab. 1 indicate the ones with a correlation coefficient $R_{\eta}$ that may appear not accurate. 


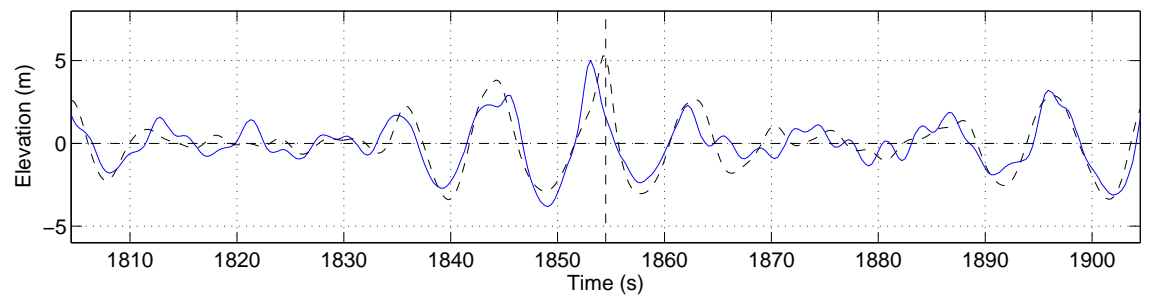

Figure 12: Comparison between reproduction and target wave in time domain. Iterative correction of phases, iteration 3. $R_{\eta}=0.84, R_{c}=0.95$

\begin{tabular}{|c|c|c|c|c|c|}
\hline Freak number & $H_{\max } / H_{s}$ & $\eta_{t_{\max }}$ & $R_{\eta}$ & $R_{c}$ & $n_{\text {iter }}$ \\
\hline $1^{*}$ & 2.4 & $5.3 \mathrm{~m}$ & 0.84 & 0.95 & 3 \\
\hline 2 & 2.3 & $5.6 \mathrm{~m}$ & 0.94 & 1.0 & 2 \\
\hline 3 & 2.2 & $5.2 \mathrm{~m}$ & 0.93 & 0.84 & 2 \\
\hline 4 & 2.6 & $7.1 \mathrm{~m}$ & 0.92 & 0.68 & 8 \\
\hline 5 & 2.3 & $4.9 \mathrm{~m}$ & 0.92 & 0.91 & 4 \\
\hline $6^{*}$ & 2.4 & $6.2 \mathrm{~m}$ & 0.85 & 0.94 & 7 \\
\hline $7^{*}$ & 2.7 & $7.6 \mathrm{~m}$ & 0.76 & 0.87 & 4 \\
\hline 8 & 2.5 & $6.7 \mathrm{~m}$ & 0.92 & 0.75 & 1 \\
\hline 9 & 2.2 & $6.0 \mathrm{~m}$ & 0.96 & 0.87 & 1 \\
\hline $10^{*}$ & 2.4 & $6.2 \mathrm{~m}$ & 0.68 & 0.84 & 2 \\
\hline
\end{tabular}

Table 1: Application of reproduction scheme on 10 different freak waves: features and accuracy estimators 
However, in those cases the comparison is accurate with respect to the crest elevation ( $R_{c}$ parameter). This has been verified that the (relative) low correlation parameter is due to a small phase shift existing between the target and reproduction signals (which are close to each other excluding this small phase shift). Consequently, the choice of $R_{\eta}$ criteria may not be optimal for all configurations but it appeared that associated to the crest amplitude ratio $R_{c}$, a compromise choice between these two criteria is efficient in the determination of an accurate reproduction of the extreme wave of interest.

Then, we can observe that in most cases the reproduction is accurate with respect to $R_{\eta}$ and $R_{c}$ and that it requires a low number of iterations for the phase correction. However, this accuracy is clearly linked to the amplitude of the extreme event and consequently to the non-linear processes involved during its creation. For the highest waves $\left(H_{\max }>2.4 H_{s}\right.$ or $\left.\eta_{\max }>1.6 H_{s}\right)$, an accurate amplitude is more difficult to achieve. Thus, this reproduction procedure have to be limited to freak waves which are not too extreme in terms of non-linearity. To this end, the two proposed criteria are efficient to assess the quality of the free surface profiles obtained. We also indicate here that the reproduction process is sensitive to the choices of parameters (duration of reproduction, location, discretization...). Furthermore, it appears to be essential to define the extreme event over a large number of frequencies. Filtering the temporal wave signal of the freak wave detected in HOS-ocean may lead to a substantial difference in the wave amplitude (see [32]). Thus, since higher frequencies are the most difficult to control in terms of phasing, it is very difficult to reproduce exactly those extreme events (and especially the amplitude of the crest). 
However, we manage to set-up a correct reproduction of several freak waves obtained by numerical simulations. They exhibit different characteristics in terms of amplitude, wave height, wave shape, etc. Next section is dedicated to the effective comparison in terms of kinematics on one example of the previously reproduced freak waves.

\subsection{Detailed comparison of freak wave and reproduced event}

In this section, once good agreement has been achieved in free surface elevation between the target and reproduced numerical rogue waves, we focus on the comparison of the two wave fields in spatial domains as well as in terms of velocities and pressure inside the fluid domain. If we are interested in the interaction of such an extreme wave with a given structure, the kinematics of the wave are of primary interest. Furthermore, these interactions may be influenced by the spatial and temporal features of the studied rogue waves.

As seen in Sec. 1, the HOS method solves the problem of wave propagation on the free surface. Volume information is consequently not directly available. Specific procedures have been developed to evaluate velocities and pressure inside the fluid domain from free surface quantities. This has been adapted from the Dirichlet to Neumann Operator (DNO) model $[6,5]$ to the HOS formalism in [17] or direct inversion of a matrix if high accuracy is required [18].

In the concern of spatial features of extreme waves, Fig. 13 presents a comparison of spatial profiles at the moment of the focusing $t_{f}$ over a domain length of $\simeq 10 \lambda_{p}$. This may be compared to Fig. 12 which presents the comparison of temporal profiles at the focusing location $x_{f}$ (on a time 
extent of $\simeq 10 T_{p}$ ).

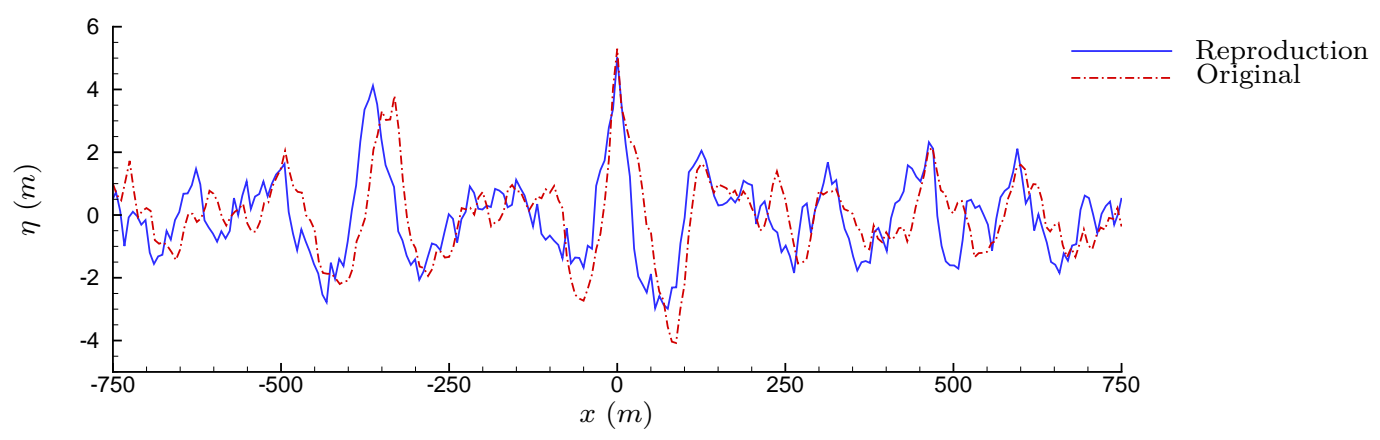

Figure 13: Comparison between reproduction and target wave in space domain. Iterative correction of phases, iteration 3. $R_{\eta}=0.84, R_{c}=0.95$

As observed for the time evolution of the free surface elevation, phasing of some high frequency components is not perfectly controlled. These are consequently present over the whole spatial domain at the moment of the focusing. However, except the presence of those small short waves, it is observed that the reproduction procedure is also accurate in the spatial domain. The main features of the original extreme event (observed at its moment of appearance) are recovered over a large spatial extent. The accuracy estimator $R_{c}$ ensures that the crest elevation is correctly reproduced.

To study in details the velocity and pressure fields induced by the rogue wave events, we compare in Fig. 14 the vertical profile of dynamic pressure and horizontal velocity of the original freak wave detected in the open domain numerical simulation and the one reproduced in the NWT. This comparison is done at the location of the freak wave crest $x_{f}$ and at the moment of 
its appearance $t_{f}$. The corresponding vertical profiles presented in Fig. 14 correspond to the case shown in Figs. 10 \&12, respectively with and without iterations.
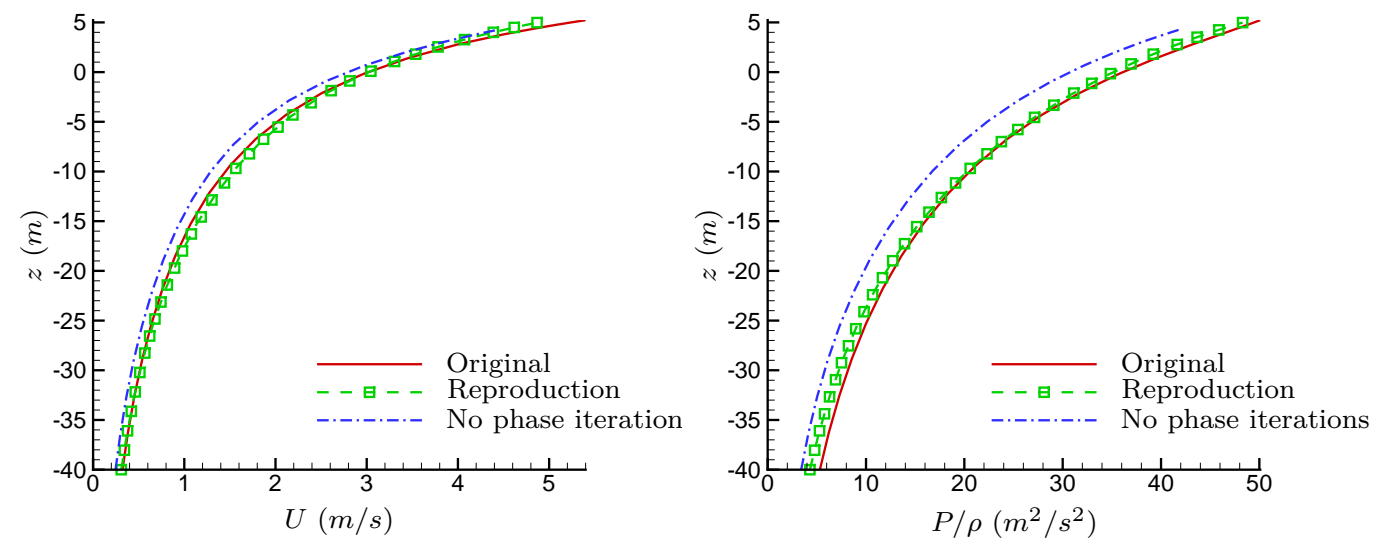

Figure 14: Comparison of vertical profiles of horizontal velocity (left) and dynamic pressure (right) between reproduction (with and without phase iterations) and target wave

This figure shows that once the free surface elevation is accurately reproduced, the vertical profile of velocity and pressure is also the same between the original freak wave and the reproduced one. Different numerical simulations run during those developments have shown that the most important factor in accurately reproducing the kinematics in the fluid domain is to have the correct amplitude of the wave. Indeed, once the free surface amplitude is correct (measured with $R_{c}$ parameter), maximum values and vertical profile of kinematic components will follow.

At the same time, without any phase iterations during reproduction process, the vertical profile of horizontal velocity is less accurate with a deficit of amplitude (that is clearly attributed to the lower crest amplitude charac- 
terized by $R_{c}=0.83$ in this case). This is associated to a dynamic pressure which is reduced compared to the original extreme event throughout the whole water column. Consequently, the slight discrepancies observed on the vertical profiles are essentially linked to the free surface amplitude of the reproduced wave.

In the end, Figs. 15, $16 \& 17$ present velocity and dynamic pressure fields at the moment of the extreme event $t_{f}$. A boundary-fitted mesh is used for the representation of these fields, for an accurate description of the domain of interest. We now restrict the field of analysis to a shorter window of $300 m\left(\simeq 2 \lambda_{p}\right)$ in the horizontal direction around the focused location $x_{f}$ and with the collocation point used during the computation. For this shorter space window, the discrepancies between the target and the reproduced rogue waves are highlighted. Since we are interested in the wave motion, we restrict the vertical domain to $z \in[-40, \eta]$ meters, although the total water depth is $h=200 \mathrm{~m}$. The same contour levels are used in both figures for ease of comparison.

It appears that the pressure and horizontal velocity fields are similar in the original 'natural' freak wave obtained with HOS-ocean and the reproduced event in HOS-NWT. The amplitude of the three fields are correctly reproduced at the crest. The two surrounding troughs however show some discrepancies in terms of location and magnitude. In addition, as observed previously, spurious high frequency components are present. This appears more clearly on the vertical velocity field which is maximum at a location other than $x_{f}$. The latter is more influenced by the accuracy on the free 

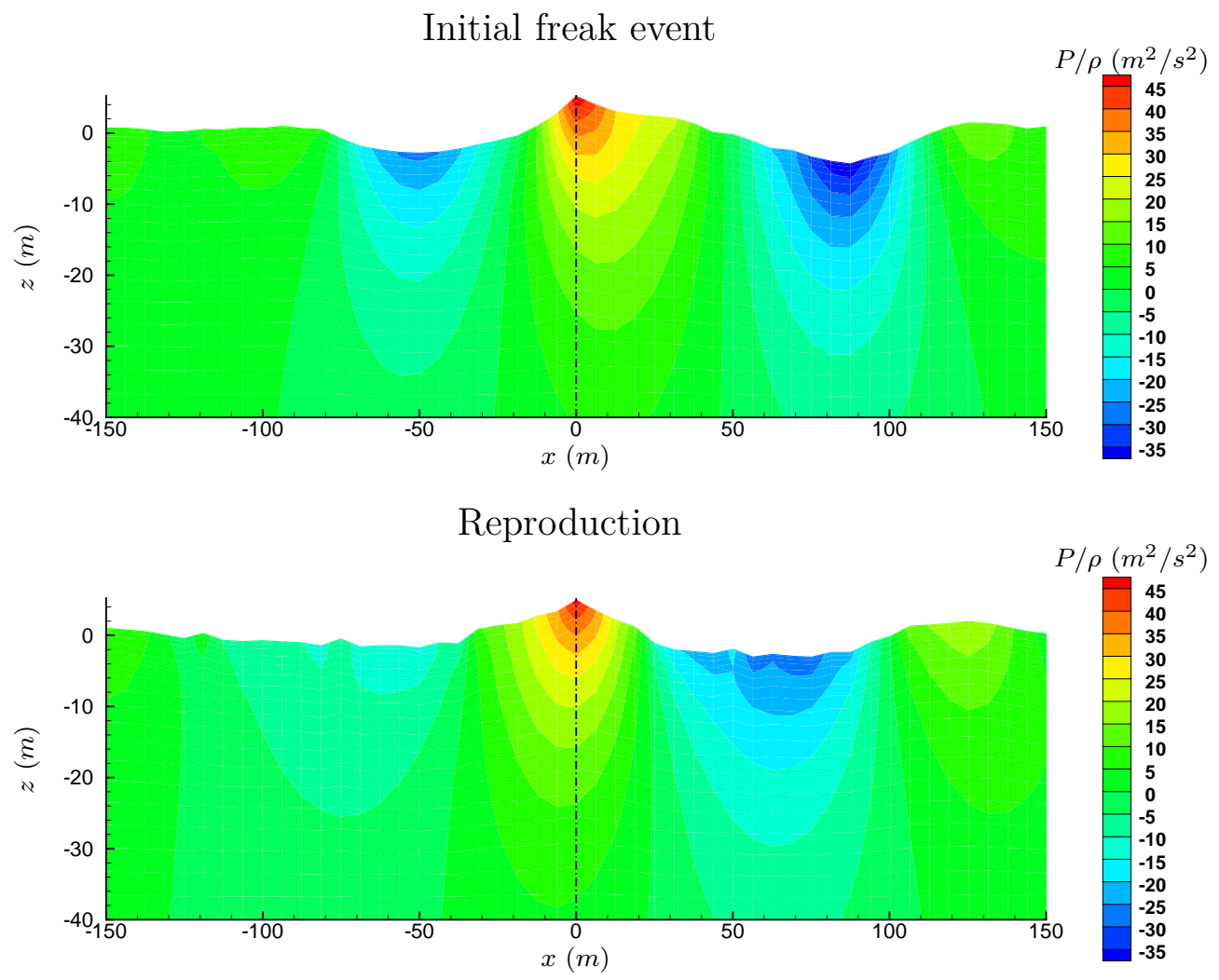

Figure 15: Dynamic pressure field of the detected freak event in periodic domain and its reproduction in a wave tank. Extreme event location is $x=0$ 

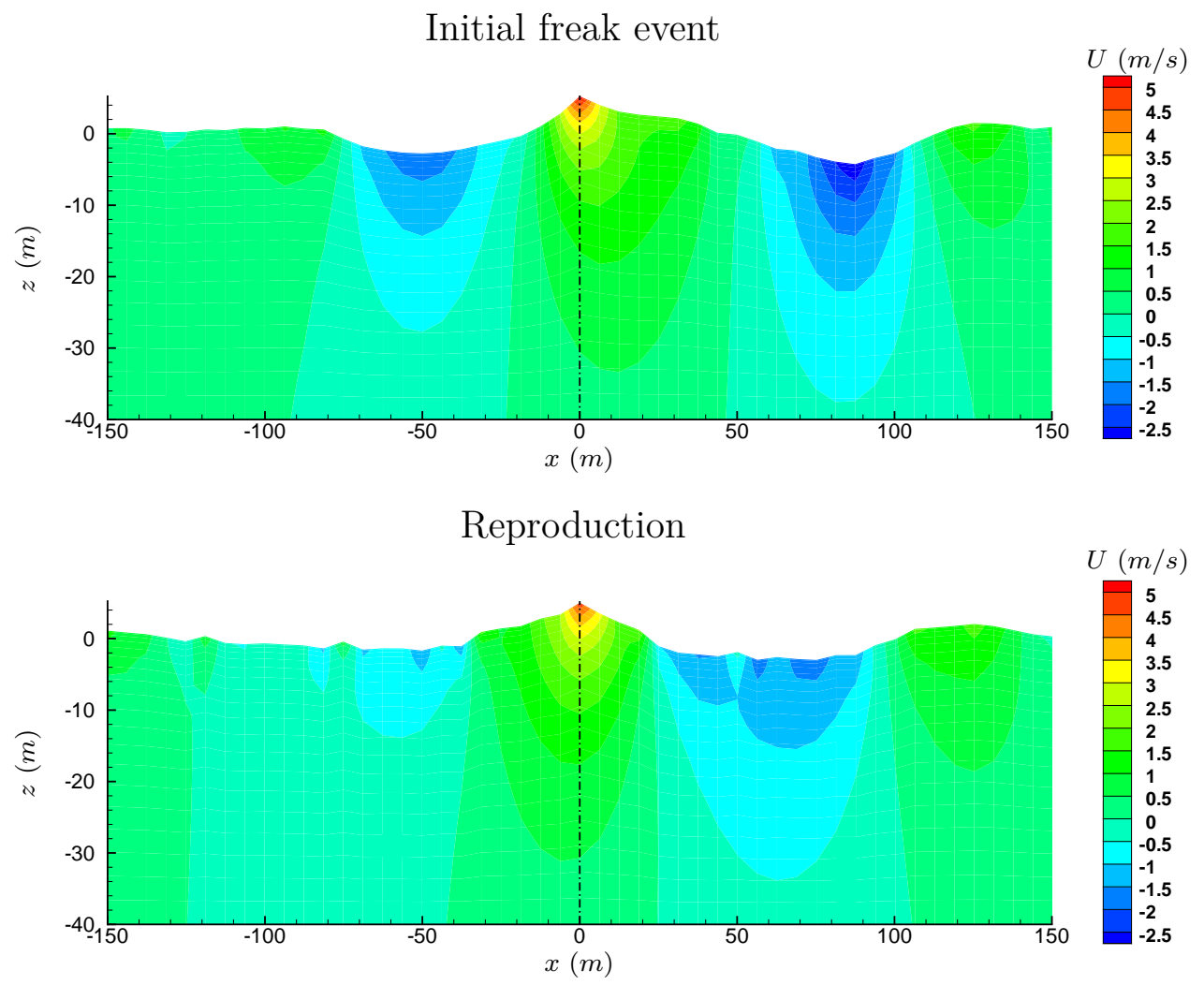

Figure 16: Horizontal velocity field of the detected freak event in periodic domain and its reproduction in a wave tank. Extreme event location is $x=0$ 

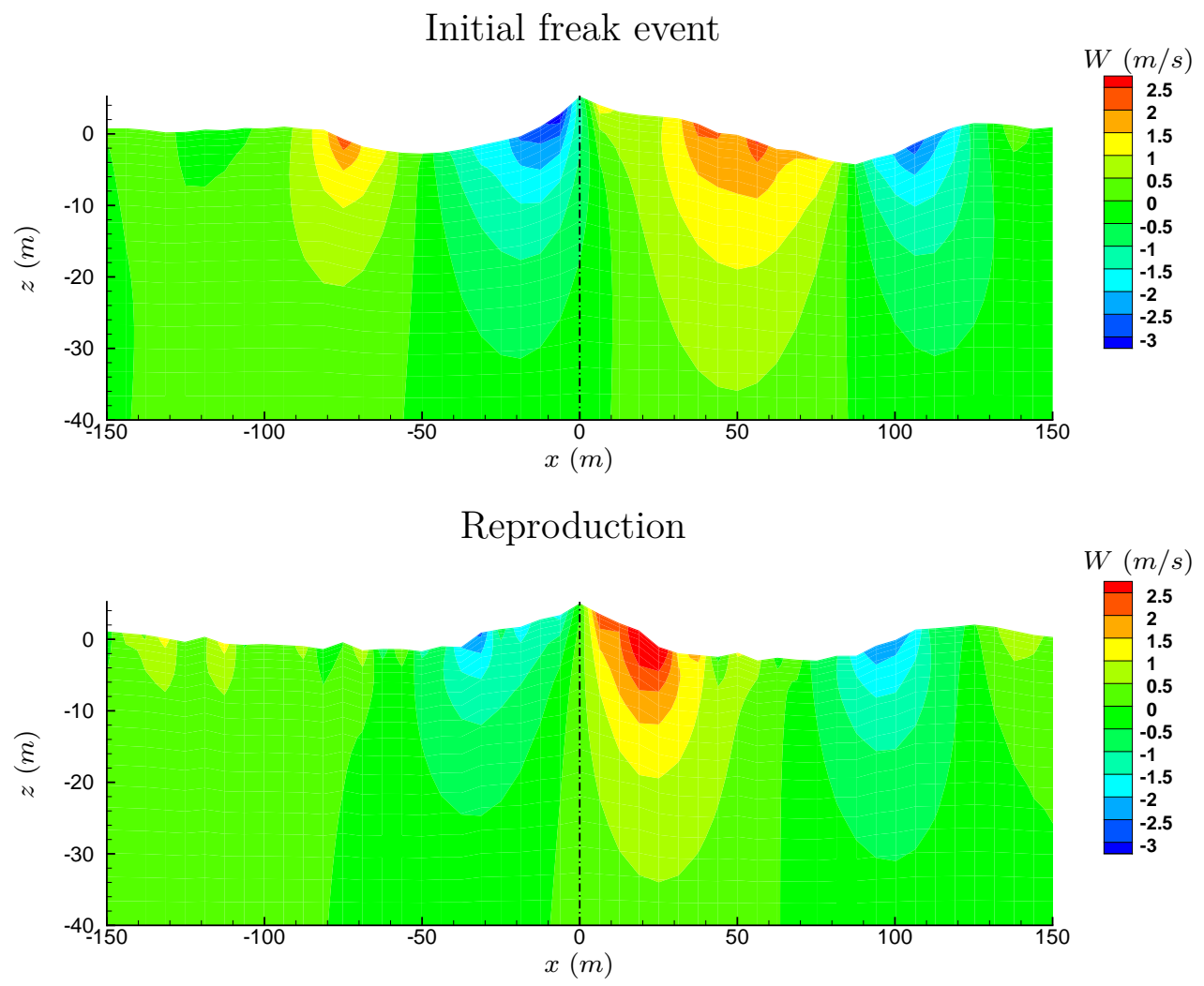

Figure 17: Vertical velocity field of the detected freak event in periodic domain and its reproduction in a wave tank. Extreme event location is $x=0$ 
surface profile in spatial domain.

Consequently, thanks to an appropriate choice of accuracy estimation through the parameters $R_{\eta}$ and $R_{c}$, it is then possible to ensure that the vertical profile under the crest of the reproduced extreme event is accurate, as is the temporal and spatial free surface elevations. The velocity and pressure fields are also similar with possible local discrepancies away from the crest.

Reminding that the HOS numerical models have been widely validated with several comparisons to experiments for free surface elevation as well as pressure fields in the fluid domain [16], this result is of particular interest for the study of extreme wave-structure interactions. The reliability of unidirectional wave basin experiments in this context of freak waves is confirmed: we have shown that if the temporal free surface elevation is correctly reproduced, the main physical phenomena at play are taken into account, resulting in the correct reproduction of fluid dynamics inside the fluid body.

\section{Conclusion \& perspectives}

This study deals with the analysis of freak waves in unidirectional wave fields and their possible reproduction in wave tanks (physical or numerical). Since we are lacking, at the present time, physical data concerning rogue waves in open ocean in terms of kinematics inside the fluid domain, this 'natural' freak wave is generated with a highly nonlinear HOS model. Such model has been widely used to study the occurrence of these extreme waves, and their performance validated. Similarly, due to the difficulty inherent in providing experimental data for the whole fluid domain in physical oceanic 
wave basins, a numerical approach has been chosen for the representation of the wave tank. Both numerical approaches (HOS-ocean and HOS-NWT) are based on the HOS formalism, but differ in the sense that one is dedicated to the simulation of an open domain condition and the other has been developed as a Numerical Wave Tank.

An efficient reproduction procedure based on single point free surface measurement has been developed. This is the most common reproduction that is needed from measurements at sea. We have chosen to reproduce the extreme event embedded in an irregular sea. The main steps are:

i) choice of focusing distance to wavemaker, $x_{f}$;

ii) choice of the frequency cut-off on input signal, $f_{\max }$;

iii) back-propagation to the wavemaker using linear theory;

iv) theoretical phase adjustment using third-order theory;

v) evaluation of wavemaker motion;

vi) iterative correction of phase shift.

The approach has been tested and validated on the New Year's wave and its ability to reproduce a freak wave with sufficient accuracy in the NWT has been demonstrated.

This new reproduction procedure was successfully applied to different freak waves detected in an HOS-ocean long-time simulation. In the latter computations, the equivalent of temporal wave probe signals of these extreme waves were extracted and used in the reproduction procedure using HOS-NWT. Temporal and spatial free surface profiles were thus obtained and a detailed comparison of the pressure and velocities field was provided 
in one case. The 'natural' freak wave simulated in the open domain was compared to the reproduced wave field in the wave basin. We observed that with the appropriate choice of procedure, it is possible to accurately reproduce the kinematics of the target extreme event in a wave tank (experimental or numerical).

This original approach, associated to the previous validations of HOS numerical models with comparisons to experiments for free surface elevation as well as pressure fields [16], ensures the correctness of the reproduction approaches in use, especially in physical wave basins. Up to now, comparisons were made only on temporal probe signals; this study overcomes this limitation.

Furthermore, this study has highlighted the potential applications of the HOS-ocean model. In the study of freak waves to date, the main interest has been in the free surface elevation, with an emphasis on understanding the physical mechanisms leading to the formation of such extreme events. However, this model may also be used to study the kinematics inside the fluid domain induced by these rogue waves in 2-D or 3-D.

The present study is limited to 2-D (i.e. one horizontal direction) since it is based on single point free surface measurement (as is often the case in open sea). However, we remind that the $3-\mathrm{D}$ character of the wavefield is essential to several non-linear processes. In future work we intend to focus on the directional effects. This may be achieved using single point measurement if in addition the directionality of the sea state at the instant of the rogue wave apparition is known. Another possibility is to use different wave probes 
around the extreme wave (an application to a 3-D focused wave packet has been reported in [16]). Furthermore, developments of SARs in 'wave mode' may offer in the near future interesting possibilities for describing 3-D free surface elevation containing extreme events. Thus, these large scale description may be used as an alternative to single point measurements.

Another prospect may be to undertake more systematic studies on the 'natural' freak wave to be reproduced. In particular, it may be interesting to investigate whether a freak wave generated by a specific physical feature, for instance a Benjamin-Feir instability, is also correctly reproduced with the procedure presented.

In conclusion, one of the principal interests in ocean engineering is the study of the interaction between extreme waves and marine structures. Using the different models and procedures developed, we are able to accurately simulate the waves, and specific developments have been made to couple HOS models of LHEEA Lab. (ECN) to Smooth Particle Hydrodynamics (SPH) model to study violent interactions (including wave breaking and free surface reconnections) [28].

\section{References}

[1] Open-source release of HOS-NWT. https://github.com/LHEEA/HOSNWT/wiki.

[2] Open-source release of HOS-ocean. https://github.com/LHEEA/HOSocean/wiki.

[3] T.A.A. Adcock, P.H. Taylor, S. Yan, Q.W. Ma, and P.A.E.M. Janssen. 
Did the Draupner wave occur in a crossing sea? Proc. R. Soc. A, 467(2134):3004-3021, 2011.

[4] Y. Agnon and H. B. Bingham. A non-periodic spectral method with applications to non linear water waves. Eur. J. Mech., B/Fluids, 18:527$534,1999$.

[5] W.J.D. Bateman, V. Katsardi, and C. Swan. Extreme ocean waves. part i. the practical application of fully nonlinear wave modelling. Appl. Ocean Res., 34(0):209 - 224, 2012.

[6] W.J.D. Bateman, C. Swan, and P.H. Taylor. On the calculation of the water particle kinematics arising in a directionnally-spread wavefield. $J$. Comp. Phys., 186:70-92, 2003.

[7] F. Bonnefoy, D. Le Touzé, and P. Ferrant. Second order directional wavemaker theory predicton and control of free waves. In Proc. $18^{\text {th }}$ Int. Workshop on Water Waves and Floating Bodies, Le Croisic, France, april 2003.

[8] J.R. Chaplin. On frequency-focusing unidirectional waves. Int. J. Offshore and Polar Engng., 6(2):131-137, 1996.

[9] G.F. Clauss and M. Klein. The new year wave: Spatial evolution of an extreme sea state. J. Offshore Mech. and Arctic Engng., 131(041001), 2009.

[10] G.F. Clauss and M. Klein. The new year wave in a seakeeping basin: Generation, propagation, kinematics and dynamics. Ocean Engng., 38(14-15):1624-1639, 2011. 
[11] G.F. Clauss and C. Schmittner. Experimental optimization of extreme wave sequences for the deterministic analysis of wave/structure interaction. J. Offshore Mech. and Arctic Engng., 129:61-67, 2007.

[12] D.G. Dommermuth. The initialization of nonlinear waves using an adjustment scheme. Wave Motion, 32:307-317, 2000.

[13] D.G. Dommermuth and D.K.P. Yue. A high-order spectral method for the study of nonlinear gravity waves. J. Fluid Mech., 184:267 - 288, 1987.

[14] G. Ducrozet, H. B. Bingham, A. P. Engsig-Karup, F. Bonnefoy, and P. Ferrant. A comparative study of two fast nonlinear free-surface water wave models. Int. J. Numer. Meth. Fluids, 69(11):1818-1834, 2012. doi: 10.1002/fld.2672.

[15] G. Ducrozet, F. Bonnefoy, D. Le Touzé, and P. Ferrant. 3-D HOS simulations of extreme waves in open seas. Nat. Hazards Earth Syst. Sci., 7(1):109-122, 2007.

[16] G. Ducrozet, F. Bonnefoy, D. Le Touzé, and P. Ferrant. A modified high-order spectral method for wavemaker modeling in a numerical wave tank. Eur. J. Mech., B/Fluids, 34:19 - 34, 2012.

[17] G. Ducrozet, D. Le Touzé, F. Bonnefoy, and P. Ferrant. Development of a fully nonlinear water wave simulator based on higher order spectral theory. In Proc. $20^{\text {th }}$ Int. Workshop on Water Waves and Floating Bodies, Longyearbayen, Norway, juin 2005. 
[18] J. D. Fenton and M. M. Rienecker. A fourier method for solving nonlinear water wave problems: application to solitary-wave interactions. $J$. Fluid Mech., 118:411-443, 1982.

[19] L. Fernandez, M. Onorato, J. Monbaliu, and A. Toffoli. Modulational instability and wave amplification in finite water depth. Nat. Hazards Earth Syst. Sci., 14:705-711, 2014. doi:10.5194/nhess-14-705-2014.

[20] H. Fernández, V. Sriram, S. Schimmels, and H. Oumeraci. Extreme wave generation using self correcting method ? revisited. Coastal Engng., $93(0): 15-31,2014$.

[21] O. Gramstad and K. Trulsen. Influence of crest and group length on the occurence of freak waves. J. Fluid Mech., 582:463-472, 2007.

[22] S. Haver. A possible freak wave event measured at the draupner jacket january 1 1995. In Proc. of Rogue Waves 2004, Brest, France, 2004. disponible à http://www.ifremer.fr/web-com/stw2004/rw/.

[23] T.B. Johannessen and C. Swan. A laboratory study of the focusing of transient and directionally spread surface water waves. Proc. R. Soc. A, 457:971-1006, 2001.

[24] C. Kharif and E. Pelinovsky. Physical mechanisms of the rogue wave phenomenon. Eur. J. Mech., B/Fluids, 22:603-634, 2003.

[25] C. Kharif, E. Pelinovsky, and A. Slunyaev. Rogue Waves in the Ocean. Springer, 2008. 
[26] Xiao-Ming Li. Ocean Surface Wave Measurement Using SAR Wave Mode Data. PhD thesis, University Hamburg, 2010.

[27] Per A. Madsen and David R. Fuhrman. Third-order theory for multidirectional irregular waves. J. Fluid Mech., 698:304-334, 52012.

[28] G. Oger, D. Le Touzé, P.M. Guilcher, and M. de Leffe. Advances in sph for naval hydrodynamics. In Proc. of the $30^{\text {th }}$ Symposium on Naval Hydrodynamics, Hobart, Tasmania, Australia, 2014.

[29] M. Onorato, S. Residori, U. Bortolozzo, A. Montina, and F.T. Arecchi. Rogue waves and their generating mechanisms in different physical contexts. Phys. Rep., 528:47-89, 2013.

[30] S. Rajendran, N. Fonseca, G.F. Guedes Soares, C. Clauss, and M. Klein. Time domain comparison with experiments for ship motions and structural loads on a container ship in abnormal waves. In Proc. of the $30^{\text {th }}$ Int. Conf. Offsh. Mech. and Arctic Engng., Rotterdam, The Netherlands, 2011. doi:10.1115/OMAE2011-50316.

[31] C. Schmittner, S. Kosleck, and J. Hennig. A phase-amplitude iteration scheme for the optimization of deterministic wave sequences. In Proc of the $28^{\text {th }}$ Int. Conf. Ocean, Offshore and Arctic Engng., Honolulu, Hawaii, USA, 2009.

[32] A. Slunyaev, E. Pelinovsky, and C. Guedes Soares. Reconstruction of extreme events through numerical simulations. J. Offshore Mech. and Arctic Engng., 136, 2014. 
[33] S. Takezawa and T. Hirayama. Advanced experimental techniques for testing ship models in transient water waves. part I: The transient test technique on ship motions in waves. In Proc. of the $11^{\text {th }}$ Symposium on Naval Hydrodynamics, pages 37-54, 1976.

[34] S. Takezawa and T. Hirayama. Advanced experimental techniques for testing ship models in transient water waves. part II: The controlled transient water waves for using in ship motion tests. In Proc. of the $11^{\text {th }}$ Symposium on Naval Hydrodynamics, pages 37-54, 1976.

[35] M. Tanaka. Verification of hasselmann's energy transfer among surface gravity waves by direct numerical simulations of primitive equations. $J$. Fluid Mech., 444:199-221, 2001.

[36] A Toffoli, E Bitner-Gregersen, M Onorato, and AV Babanin. Wave crest and trough distributions in a broad-banded directional wave field. Ocean Engng., 35(17):1784-1792, 2008.

[37] A. Toffoli, L. Fernandez, J. Monbaliu, M. Benoit, E. Gagnaire-Renou, J. M. Lefèvre, L. Cavaleri, D. Proment, C. Pakozdi, C. T. Stansberg, T. Waseda, and M. Onorato. Experimental evidence of the modulation of a plane wave to oblique perturbations and generation of rogue waves in finite water depth. Phys. of Fluids, 25(091701), 2013.

[38] A. Toffoli, O. Gramstad, K. Trulsen, J. Monbaliu, E. M. BitnerGregersen, and M. Onorato. Evolution of weakly nonlinear random directional waves: laboratory experiments and numerical simulations. J. Fluid Mech., 664:313-336, 2010. 
[39] A. Toffoli, M. Onorato, E. M. Bitner-Gregersen, and J. Monbaliu. Development of a bimodal structure in ocean wave spectra. J. Geophys. Res., 115(C03006), 2010. doi:10.1029/2009JC005495.

[40] K. Trulsen. Simulating the spatial evolution of a measured time series of a freak wave. In Proc. of the Workshop 'Rogue Waves 2000', pages 265-274, Brest, France.

[41] T. Waseda, T. Kinoshita, and H. Tamura. Evolution of a random directional wave and freak wave occurrence. J. Phys. Oceanogr., 39:621-639, 2009.

[42] B. J. West, K. A. Brueckner, R. S. Janda, M. Milder, and R. L. Milton. A new numerical method for surface hydrodynamics. J. Geophys. Res., 92:11803 - 11824, 1987.

[43] W. Xiao, Y. Liu, G. Wu, and D.K.P. Yue. Rogue wave occurence and dynamics by direct simulations of nonlinear wave-field evolution. $J$. Fluid Mech., 720:357-392, 2013.

[44] V. Zakharov. Stability of periodic waves of finite amplitude on the surface of a deep fluid. J. Appl. Mech. Tech. Phys., pages 190-194, 1968.

[45] H.D. Zhang, C. Guedes Soares, and M. Onorato. Modelling of the spatial evolution of extreme laboratory wave heights with the nonlinear schrödinger and dysthe equations. Ocean Engng., 89(0):1 - 9, 2014. 